Lipidsenker mit Zusatzeffekt

\section{Beugen Statine einem Prostatakarzinom vor?}

— Die Vermutung, dass Statine einem Prostatakarzinom vorbeugen können, erhält Unterstützung durch eine neue Studie [Nelly T et al. J Urol 2011; 186: 86-90]. In der retrospektiven Untersuchung wurden die Prostatabioptate von 4.204 Männern verglichen, davon waren 1.022 Statinanwender. Alle Männer hatten sich wegen einer positiven digital-rektalen Untersuchung oder wegen erhöhter PSA-Werte einer Prostatabiopsie unterzogen.

Hierbei war bei 55,2\% der Männer unter Statintherapie (mittleres Alter 65,7 Jahre) ein Prostatakarzinom entdeckt worden. Von den Männern ohne Statin (mittleres Alter 63,5 Jahre) hatten 57,8\% ein Prostatakarzinom $(p=0,15)$. Auch wenn ein Prostatakarzinom diagnostiziert wurde, waren Statinanwender im Vorteil gegenüber Nicht-Anwendern. Sie hatten nämlich seltener einen positiven Befund bei der digital-rektalen Untersuchung ( $53 \%$ vs. $8,9 \%, p<0,01)$, seltener einen Gleason-Score von 7 oder mehr $(61,4 \%$ vs. $72,4 \%, p=0,02)$ sowie seltener ein ausgedehntes Prostatakarzinom mit mindestens drei positiven Stanzen $(27,2 \%$ vs. $31,4 \%, p<0,01)$.

Die Schutzwirkung der Statintherapie blieb auch erhalten, wenn für Risikofaktoren wie Alter, BMI oder Prostatavolumen adjustiert wurde. Nach der multivariaten Analyse hatten Männer unter einem Statin seltener generell ein Prostatakarzinom $(R R=0,92)$ und seltener ein hochgradiges $(R R=0,76)$ oder ein großvolumiges Prostatakarzinom ( $R R=0,86$ ).

Ähnlich positive Auswirkungen einer Statintherapie auf Prostatakarzinome wurden auch in früheren Studien teilweise beobachtet. Als einen möglichen Mechanismus nennen die Studienautoren die Absenkung des PSA-Spiegels unter einer Statintherapie. Dabei weisen sie darauf hin, dass nicht zu befürchten stehe, dieser Effekt könne die Entdeckung eines Prostatakarzinoms bei Statinanwendern erschweren. Wenn ein Prostatakarzinom bestehe, seien die PSAWerte ebenso hoch wie bei Nicht-Statinanwendern - dies habe sich in ihrer Studie gezeigt.

Dr. Beate Schumacher

\section{Wo sich MRSA wohlfühlt}

\section{Killerkeime in der Kitteltasche}

\begin{abstract}
- Sechs von zehn Arztkitteln beherbergen pathogene Keime, darunter auch multiresistente Vertreter wie Methicillinresistente Staphylokokken (MRSA). Das hat eine Studie ergeben, in der Abstriche von den Bauchpartien, den Ärmelenden und den Kitteltaschen genommen worden waren [Wiener-Well Y et al. Am J Infect Control 2011; 39: 555-9]. An der Studie hatten sich 75 Krankenschwestern und 60 Ärzte beteiligt. Bei $63 \%$ aller Studienteilnehmer (Schwestern 65\%, Ärzte $60 \%$ ) war mindestens ein Abstrich positiv auf potenziell pathogene Keime. Als einziger Risikofaktor konnte die Anzahl der Kittelwechsel ermittelt werden. Die hohe Prävalenz kontaminierter Arbeitskleidung rühre womöglich von mangelnder Handhygiene her, meinen die Mikrobiologen. Resistente Keime fanden sich nämlich vor allem an den Taschen und Ärmelenden.

Dr. Robert Bublak
\end{abstract}

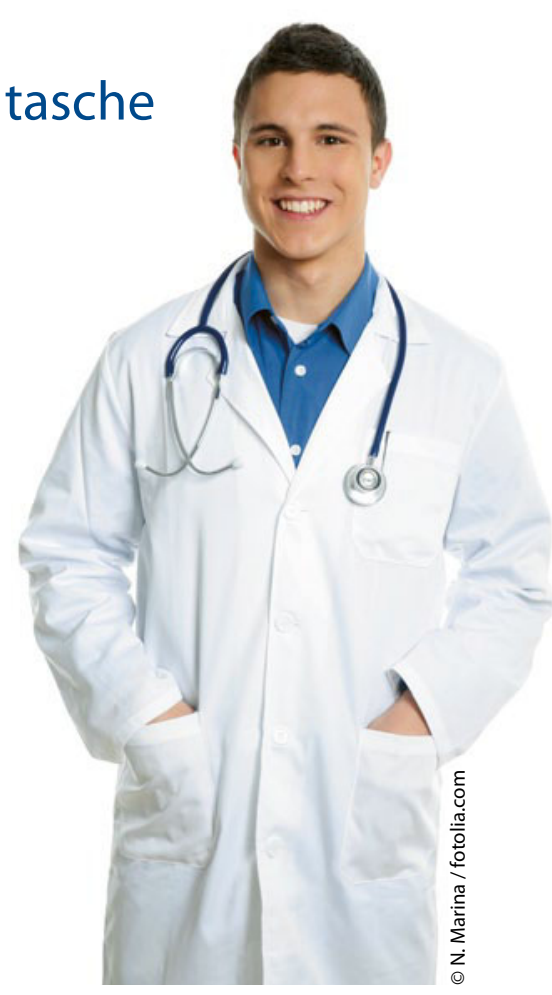

Ist er ein Taschenkeimträger?

\title{
Zystektomie
}

\section{Drei Faktoren lassen den Blasenkrebs wieder wachsen}

— Die Wahrscheinlichkeit, dass nach radikaler Zystektomie ein urotheliales Karzinom rezidiviert, ist für Patienten höher, bei denen die Prostata mitbetroffen ist, das Karzinom multifokal in der Blase auftritt und bei denen nach Zystektomie ein orthotoper Blasenersatz angelegt wird. Das ist das Ergebnis einer aktuellen US-amerikanischen Studie mit insgesamt 1.506 Patienten, die sich einer radikalen Zystektomie unterziehen mussten und im Durchschnitt über 13,5 Jahre lang überwacht wurden. 85 Patienten $(5,6 \%)$ erlitten ein Rezidiv, durchschnittlich 13,3 Monate nach der Zystektomie. 80 dieser Patienten war eine kutane Harnableitung angelegt worden (insgesamt wurden 1.243 so versorgt, $6,4 \%$ ), fünf hatten eine Neoblase bekommen (insgesamt $242 ; 2,1 \%$ ). Als Rezidiv-Risikofaktoren kristallisierten sich Miteinbeziehung der Prostata (Hazard Ratio [HR]: 4,89; $\mathrm{p}<0,0001$ ), Multifokalität des primären Blasenkarzinoms (HR: 2,34; $\mathrm{p}$ $=0,0001$ ) und orthotoper Blasenersatz (HR: 0,34; $p=0,02$ ) heraus.

Das Fünf-Jahres-Überleben wiederum hing entscheidend davon ab, ob das Rezidiv bei Diagnose schon symptomatisch oder noch asymptomatisch war. $80 \%$ der asymptomatischen Patienten, deren Rezidiv zytologisch diagnostiziert wurde, lebten auch noch nach fünf Jahren, hingegen nur $41 \%$ der Rezidivpatienten mit Beschwerden $(p<0,0001)$. Auch wenn ein Blasenkarzinomrezidiv nach radikaler Zystektomie eher selten vorkommt $(5,6 \%)$, müsse mehr Wert auf die regelmäßige Nachsorge dieser Patienten gelegt werden, so die Autoren. Ziel müsse es sein, Rezidive zu erkennen, solange sie noch asymptomatisch sind. Nur dann hätten die Patienten eine gute Überlebenschance.

Dr. Dagmar Kraus 